\title{
Precise tests of chiral perturbation theory from Ke4 decays by the NA48/2 experiment
}

\author{
Brigitte Bloch-Devaux* ${ }^{*}$ \\ IRFU/SPP, CEA-Saclay \\ E-mail: brigitte.bloch@cea.fr
}

\begin{abstract}
Very large samples of charged kaon decays have been accumulated in 2003-2004 by the NA48/2 experiment at the CERN SPS. Among many rare decay modes, the analysis of $\mathrm{K}^{ \pm} \rightarrow \pi^{+} \pi^{-} \mathrm{e}^{ \pm} v$ $\left(\mathrm{K}_{\mathrm{e} 4}\right)$ decays allows a quasi model-independent approach to the study of low energy $\pi \pi$ scattering. From a data sample of more than 1 million $\mathrm{K}_{\mathrm{e} 4}$ decays, the form factors and their energy dependence are simultaneously measured with an improved precision. From the evolution of the $\pi \pi$ phsase shift close to threshold, scattering lengths can be extracted with an experimental precision of few percents, providing an accurate test of Chiral Perturbation Theory predictions. This result can be combined with the independent but complementary NA48/2 result obtained in the analysis of 60 millions $\mathrm{K}^{ \pm} \rightarrow \pi^{0} \pi^{0} \pi^{ \pm}\left(\mathrm{K}_{3 \pi}\right)$, leading to an experimental measurement of $a_{0}$ and $a_{2}$, the isospin 0 and $2 \mathrm{~S}$-wave $\pi \pi$ scattering lengths, of unprecedented precision.
\end{abstract}

6th International Workshop on Chiral Dynamics, CD09

July 6-10, 2009

Bern, Switzerland

\footnotetext{
* Speaker.

†n behalf of the NA48/2 collaboration: Cambridge, CERN, Chicago, Dubna, Edinburgh, Ferrara, Florence, Mainz, Northwestern, Perugia, Pisa, Saclay, Siegen, Turin, Vienna
} 


\section{Introduction}

In the past years, $\mathrm{K}^{ \pm} \rightarrow \pi^{+} \pi^{-} \mathrm{e}^{ \pm} \boldsymbol{V}$ decays $\left(\mathrm{K}_{\mathrm{e} 4}\right)$ were traditionally the cleanest laboratory to study $\pi \pi$ scattering close to threshold and extract the values of the S-wave scattering lengths. Three experiments have collected sizable samples of $\mathrm{K}_{\mathrm{e} 4}$ decays: the NA48/2 collaboration at the CERN/SPS has already published results based on a partial sample of $670000 \mathrm{~K}^{ \pm}$decays [1], the E865 collaboration at BNL analyzed about 400000 such $\mathrm{K}^{+}$decays [2] and the Geneva-Saclay collaboration (S118) at the CERN/PS $30000 \mathrm{~K}^{+}$decays [3].

The NA48/2 collaboration at the CERN/SPS has now analyzed the total available statistics collected in 2003-2004, more than one million decays in both charge modes. Results based on the full data sample (1.13 million $\mathrm{K}_{\mathrm{e} 4}$ decays) will be presented here. The form factors and $\pi \pi$ phase shift which characterize the $\mathrm{K}_{\mathrm{e} 4}$ decay are measured simultaneously in independent bins of $M_{\pi \pi}$ allowing to study their behavior close to threshold without relying on any specific modeling. Swave $\pi \pi$ scattering lengths are then extracted with an improved precision using recent theoretical work [ [

\section{Experimental setup}

Two simultaneous intense $\mathrm{K}^{+}$and $\mathrm{K}^{-}$beams were produced by $400 \mathrm{GeV} / c$ primary CERN/SPS protons, impinging on a beryllium target. The beams were then deflected in a front-end achromat to select momenta in the range $(60 \pm 3) \mathrm{GeV} / c$ and focused at $\sim 200 \mathrm{~m}$ downstream in front of the first spectrometer chamber. A schematic view of the beam line and detector can be found in Ref. [5]. At the entrance of the decay volume, a $114 \mathrm{~m}$ long vacuum tank, the kaon beams contain $\sim 2.3 \times 10^{6} \mathrm{~K}^{+}$and $\sim 1.3 \times 10^{6} \mathrm{~K}^{-}$per pulse of $4.5 \mathrm{~s}$ duration leading to a flux ratio $\mathrm{K}^{+} / \mathrm{K}^{-}$close to 1.8. The NA48 detector and its performances are described with full details elsewhere [6].

The main components used in the $\mathrm{K}_{\mathrm{e} 4}$ analysis are:

- a magnetic spectrometer consisting of a dipole magnet surrounded by two sets of drift chambers achieving a momentum resolution $\sigma(p) / p=(1.02 \oplus 0.044 p) \%(p$ in $\mathrm{GeV} / c)$.

- a 27 radiation length liquid krypton calorimeter used to measure electromagnetic deposits and identify electrons through their $E / p$ ratio. The transverse segmentation into 13248 projective cells gives an energy resolution $\sigma(E) / E=(3.2 / \sqrt{E} \oplus 9.0 / E \oplus 0.42) \%(\mathrm{E}$ in $\mathrm{GeV})$ and a space resolution for isolated showers $\sigma_{x}=\sigma_{y}=(0.42 / \sqrt{E} \oplus 0.06) \mathrm{cm}$.

- a two plane segmented scintillator hodoscope allowing to trigger the detector readout on charged track topologies with a time resolution $\sim 150 \mathrm{ps}$.

- a two-level trigger logic selecting events with at least 3 tracks coming from a common vertex.

\section{The $\mathrm{K}_{\mathrm{e} 4}$ decay analysis}

The data were selected for three track vertex topologies, requiring two opposite sign pions and one $\mathrm{e}^{+}\left(\mathrm{e}^{-}\right)$identified from its $E / p$ ratio, defining a Right Sign (RS) signal combination. The reconstructed 3-track invariant mass (assigning a pion mass to each track) and $p_{t}$ relative to the beam axis had to be outside an ellipse centered on the kaon mass and zero $p_{t}$, with semi-axes $\pm 20 \mathrm{MeV} / c^{2}$ and $\pm 35 \mathrm{MeV} / c$, allowing missing energy and $p_{t}$ for the neutrino. No more than $3 \mathrm{GeV}$ energy 
deposits in the calorimeter, non associated to tracks but in-time with the considered track combination, were allowed, keeping only a limited amount of soft radiative decays. The background sources are $\mathrm{K}^{ \pm} \rightarrow \pi^{+} \pi^{-} \pi^{ \pm}$decays with subsequent $\pi \rightarrow \mathrm{e} v$ decay or a pion misidentified as an electron, and $\mathrm{K}^{ \pm} \rightarrow \pi^{ \pm} \pi^{0}\left(\pi^{0}\right)$ decays with subsequent Dalitz decay of a $\pi^{0}$ to $\mathrm{e}^{+} \mathrm{e}^{-} \gamma$ with an electron misidentified as a pion and photon(s) undetected. Wrong Sign events (WS), $\mathrm{K}^{ \pm} \rightarrow \pi^{+} \pi^{+} \mathrm{e}^{\mp} v$, are strongly disfavored as violating the $\Delta \mathrm{S}=\Delta \mathrm{Q}$ rule. Therefore they bring a direct control of the background events. The relative level of background/signal events is $\sim 0.5 \%$ and has been crosschecked using Monte Carlo simulation of the contributing background processes. This is illustrated in Figure 1-left by the distribution of the reconstructed beam momentum under the assumption of a missing neutrino.

\subsection{Kinematics and Form Factor analysis procedure}

The $\mathrm{K}_{\mathrm{e} 4}$ decay is fully described by the five kinematic Cabibbo-Maksymowicz variables [7]: two invariant masses squared $S_{\pi}\left(=M_{\pi \pi}^{2}\right)$ and $S_{\mathrm{e}}\left(=M_{\mathrm{ev}}^{2}\right)$ and three angles $\theta_{\pi}, \theta_{e}$ and $\Phi$ as shown in Figure 1-right.

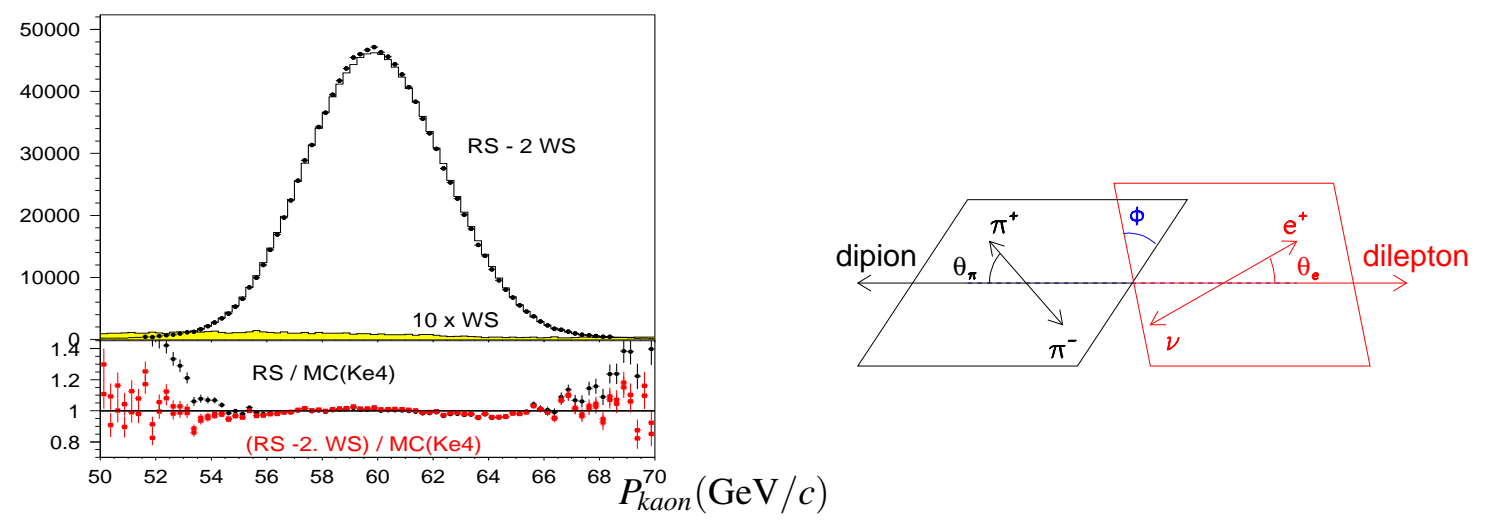

Figure 1: Left: Distribution of the reconstructed beam momentum $(\mathrm{GeV} / c)$. Data (background subtracted) are shown as symbols with error bars, simulation as open histogram and background (increased by a factor of 10 to be visible) from wrong sign events as shaded area. The insert shows the ratio of Data and simulated distributions before and after background subtraction. Right: Topology of the charged $\mathrm{K}_{\mathrm{e} 4}$ decay showing the angle definitions in the dipion (dilepton) rest frame and between the two rest frames.

Three axial $(F, G, R)$ and one vector $(H)$ complex form factors contribute to the transition amplitude expressed in terms of four combinations $(F 1, F 2, F 3, F 4)$ which can be then developed in a partial wave expansion of S, P-waves, identified with the phases of the $\pi \pi$ scattering [8] under the assumption of $\mathrm{T}$ invariance:

$$
F=F_{s} e^{i \delta_{s}}+F_{p} e^{i \delta_{p}} \cos \theta_{\pi}, \quad G=G_{p} e^{i \delta_{g}}, \quad H=H_{p} e^{i \delta_{h}}
$$

The third axial form factor $R$ is suppressed by a factor $m_{\mathrm{e}}^{2} / S_{\mathrm{e}}$ and consequently, cannot be measured in $\mathrm{K}_{\mathrm{e} 4}$ decays. Assuming the same phase for $F_{p}, G_{p}, H_{p}$, only one phase difference and four real form factors are left $\left(\delta=\delta_{s}-\delta_{p}\right.$, and $\left.F_{s}, F_{p}, G_{p}, H_{p}\right)$.

The event sample is then distributed over a grid of equal population boxes in the five-variable space. The chosen grid has ten bins in $M_{\pi \pi}$, five bins in $M_{\mathrm{e} v}$, five bins in $\cos \theta_{\pi}$, five bins in $\cos \theta_{\mathrm{e}}$ and twelve bins in $\phi$, i.e. a total of 15000 five-dimensional boxes. Using the total sample of $726000 \mathrm{~K}^{+}\left(404000 \mathrm{~K}^{-}\right)$decays, there are $48 \mathrm{~K}^{+}\left(27 \mathrm{~K}^{-}\right)$per box. The Monte-Carlo simulation 
sample, 25 times larger than the data sample, takes into account acceptance, resolution effects as well as time-dependence of the beam geometry and experimental conditions. It also includes some of the electromagnetic effects, namely the classical Coulomb attraction between the two charged pions and the emission of real photon(s) as described by the PHOTOS generator [ [ the simulation.

In this analysis, the branching fraction is not measured, so only relative form factors are accessible: $F_{p} / F_{s}, G_{p} / F_{s}, H_{p} / F_{s}$ and the phase shift $\delta$. Without prior assumption on the shape of their variation with $M_{\pi \pi}$, the form factors and phase shift are measured in the independent $M_{\pi \pi}$ bins and do not depend upon any particular model. At a second stage of the analysis, the observed variations of the form factors and phase shift with $M_{\pi \pi}$ are used to determine other parameter values through specific models. Under the assumption of isospin symmetry, the form factors can be developed in a series expansion of the dimensionless invariants $q^{2}\left(q^{2}=\left(S_{\pi} / 4 m_{\pi}^{2}\right)-1\right)$ and $S_{\mathrm{e}} / 4 m_{\pi}^{2}$.

The $F_{s}$ form factor variation is described with three "slope" terms:

$$
F_{s}=f_{s}\left(1+f_{s}^{\prime} / f_{s} q^{2}+f_{s}^{\prime \prime} / f_{s} q^{4}+f_{\mathrm{e}}^{\prime} / f_{s} S_{\mathrm{e}} / 4 m_{\pi}^{2}\right),
$$

while two (constant and slope) terms are enough to describe the $G_{p}$ form factor:

$$
G_{p} / f_{s}=g_{p} / f_{s}+g_{p}^{\prime} / f_{s} q^{2},
$$

and constants terms to describe the $F_{p}$ and $H_{p}$ form factors. The numerical results for all terms are given below:

$$
\begin{array}{lr}
f_{s}^{\prime} / f_{s}=0.152 \pm 0.007_{\text {stat }} \pm 0.005_{\text {syst }} \\
f_{s}^{\prime \prime} / f_{s}=-0.073 \pm 0.007_{\text {stat }} \pm 0.006_{\text {syst }} \\
f_{\mathrm{e}}^{\prime} / f_{s}=0.068 \pm 0.006_{\text {stat }} \pm 0.007_{\text {syst }} \\
f_{p} / f_{s}=-0.048 \pm 0.003_{\text {stat }} \pm 0.004_{\text {syst }} \\
g_{p} / f_{s}=0.868 \pm 0.010_{\text {stat }} \pm 0.010_{\text {syst }} \\
g_{p}^{\prime} / f_{s}=0.089 \pm 0.017_{\text {stat }} \pm 0.013_{\text {syst }} \\
h_{p} / f_{s}=-0.398 \pm 0.015_{\text {stat }} \pm 0.008_{\text {syst }}
\end{array}
$$

The overall agreement between data and simulated distributions is excellent for each of the five kinematic variable projections. as can be seen from Figure 2. The systematic errors quoted are mainly due to background and acceptance uncertainties. They have been revisited compared to [1], including a restricted range [54.,66.] $\mathrm{GeV} / c$ of the reconstructed kaon momentum in the selection to minimize the background contamination (see Figure 1-left) and thus its contribution to the systematic uncertainties.

\subsection{Determination of the scattering lengths}

To extract scattering length values from the phase shift measurements of $\left(\delta=\delta_{s}-\delta_{p}\right)$, more theoretical ingredients are needed. The Roy equations [10], based on analyticity, unitary and crossing symmetries, allow to predict the $\pi \pi$ phase values close to threshold using experimental measurements above the matching point $(\mathrm{E}=0.8 \mathrm{GeV})$ and two subtraction constants, $a_{0}$ and $a_{2}$, the isospin 0 and $2 \mathrm{~S}$-wave scattering lengths (in units of $m_{\pi^{+}}$). Conversely, from measurements of the phases and using the Roy equations, one can determine the corresponding values of the subtraction constants. Numerical solutions of the Roy equations have been developed by several groups [11] 

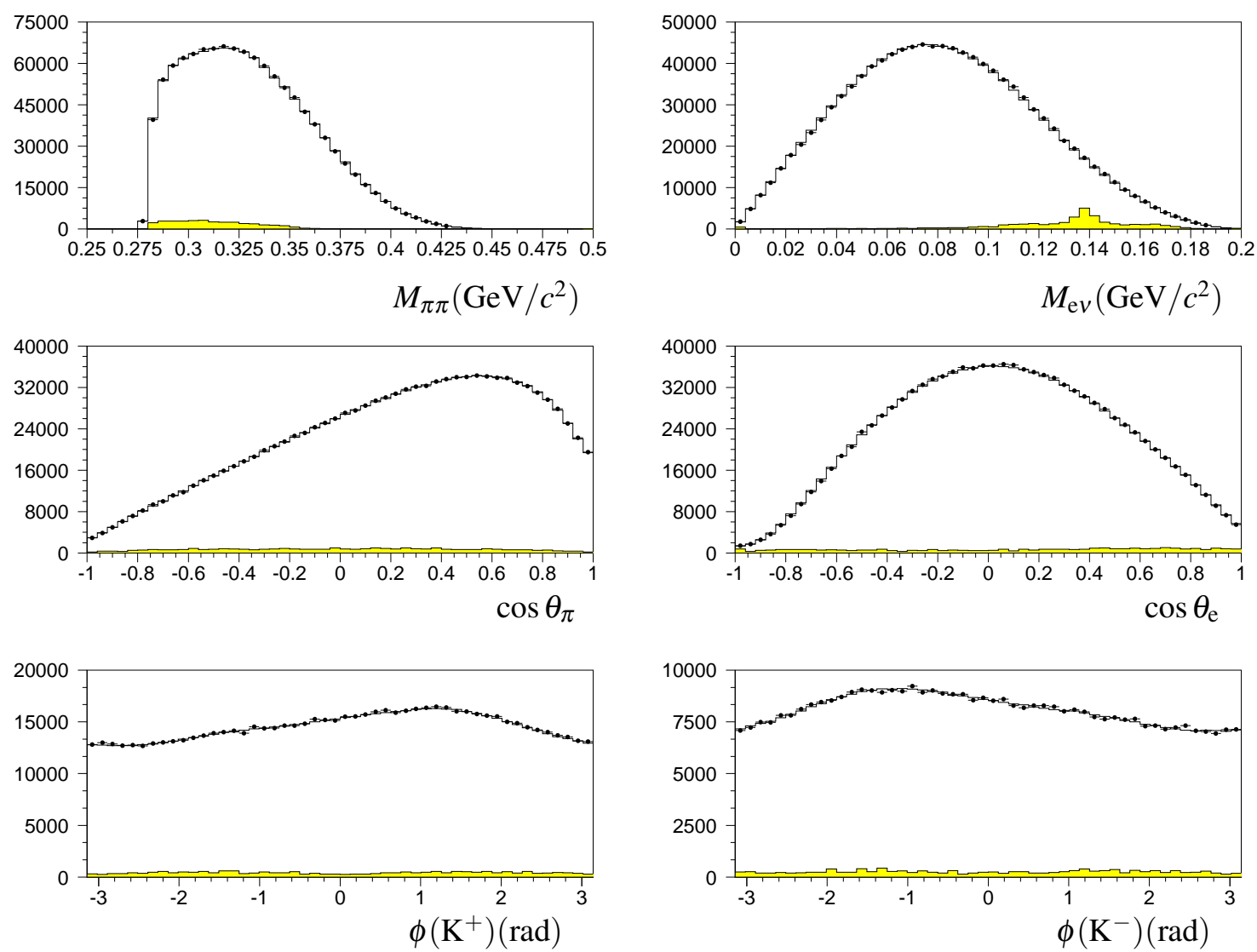

Figure 2: Distributions of the five Cabibbo-Maksymowicz variables as observed in the detector. The $\phi$ distributions are shown separately for $\mathrm{K}^{+}$and $\mathrm{K}^{-}$to emphasize their opposite behavior. Data (after background subtraction) are shown as symbols with error bars, simulation using the fitted form factor values as histograms. The background contribution (WS events), superimposed as shaded area, is increased by a factor of 10 to be visible on the linear scale.

and can be used for that purpose. Recent theoretical work, triggered by NA48/2 early results, has shown that isospin symmetry breaking may also alter the phases measured in the $\mathrm{K}_{\mathrm{e} 4}$ decays when all mass effects $\left(m_{\pi^{+}} \neq m_{\pi^{0}}, m_{u} \neq m_{d}\right)$, neglected so far in previous analyzes, are considered [П]. Even if evaluated as a modest correction in term of absolute magnitude (10 to $15 \mathrm{mrad}$ ) over the whole range of $M_{\pi \pi}$, the coherent shift toward higher values of the phases has non negligible implications when extracting scattering lengths from such measurements. Figure 3 -left shows all experimental phase measurements from $\mathrm{K}_{\mathrm{e} 4}$ data after subtraction of the isospin mass effects. The impact of the correction on the very precise measurements from NA48/2 is illustrated in Figure 3 -right. The simultaneous determination of both scattering lengths $a_{0}$ and $a_{2}$ translates into a $68 \% \mathrm{CL}$ contour in the $\left(a_{0}, a_{2}\right)$ plane. The wide Universal Band corresponds to the range of allowed solutions given the experimental inputs at high energy, while the narrow band corresponds to an additional constraint from Chiral Perturbation Theory (ChPT) [12]. The NA48/2 experimental phase measurements translate in a 2-parameter fit as: 


$$
\begin{aligned}
& a_{0}=0.2220 \pm 0.0128_{\text {stat }} \pm 0.0050_{\text {syst }} \pm 0.0037_{\text {theo }} \\
& a_{2}=-0.0432 \pm 0.0086_{\text {stat }} \pm 0.0034_{\text {syst }} \pm 0.0028_{\text {theo }}
\end{aligned}
$$

with a $97 \%$ correlation coefficient. The theoretical error has been estimated in this 2-parameter fit following the prescription described in [4] and is dominated by the experimental precision of the inputs to the Roy equation $\left(a_{2}\right)$ and the neglected Higher Order terms when introducing the mass effects $\left(a_{0}\right)$. Using the additional ChPT constraint $\left(a_{2}=-0.0444+0.236\left(a_{0}-0.22\right)-0.61\left(a_{0}-\right.\right.$ $\left.0.22)^{2}-9.9\left(a_{0}-0.22\right)^{3} \pm 0.0008\right)$, the value from the 1-parameter fit is:

$$
a_{0}=0.2206 \pm 0.0049_{\text {stat }} \pm 0.0018_{\text {syst }} \pm 0.0064_{\text {theo }}
$$

This result can be compared to the most precise prediction of ChPT [12]:

$$
a_{0}=0.220 \pm 0.005_{\text {theo }}, a_{2}=-0.0444 \pm 0.0010_{\text {theo }}
$$
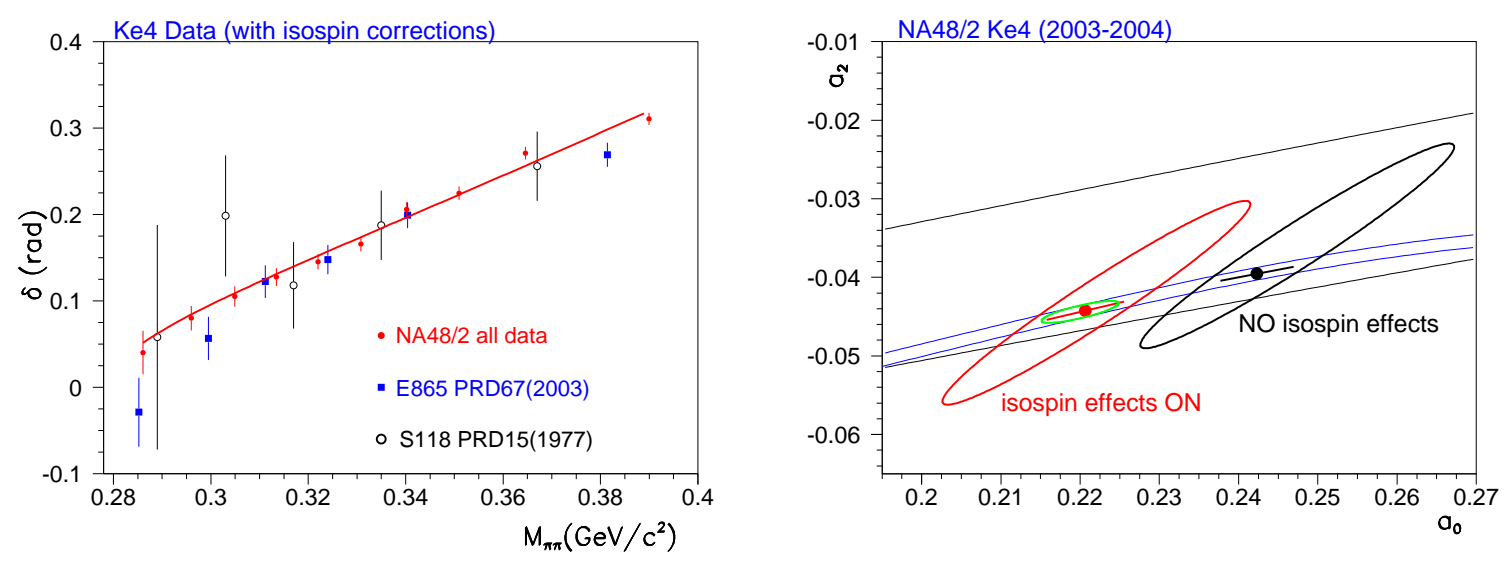

Figure 3: Left: Phase shift $(\delta)$ measurements with isospin mass effects included for all $\mathrm{K}_{\mathrm{e} 4}$ available results. The line corresponds to the 2-parameter fit of the NA48/2 data alone. Right: Fits of the NA48/2 $\mathrm{K}_{\mathrm{e} 4}$ data in the $\left(a_{0}, a_{2}\right)$ plane without (black) and with (red) isospin mass effects. Errors are statistical only. Ellipses are $68 \%$ CL contours and symbols are the result of the one-parameter fit imposing the ChPT constraint. The small (green) ellipse corresponds to the prediction from ChPT.

\section{Combination of NA48/2 results}

The scattering length results from the analysis of the cusp effect observed in $\mathrm{K}^{ \pm} \rightarrow \pi^{0} \pi^{0} \pi^{ \pm}$ decays have been reported at this conference [13]. The $\mathrm{K}_{\mathrm{e} 4}$ and cusp results are statistically independent, have very different systematic uncertainties (background and particle identification in one case, control of calorimetry and neutral trigger in the other) and show different correlations in the $\left(a_{0}, a_{2}\right)$ plane:

$$
\begin{array}{rcc} 
& \mathrm{K}_{\mathrm{e} 4} & \mathrm{~K}_{3 \pi} \text { cusp } \\
a_{0}= & 0.2220 \pm 0.0128_{\text {stat }} \pm 0.0050_{\text {syst }} & \\
a_{0}-a_{2}= & & 0.2571 \pm 0.0048_{\text {stat }} \pm 0.0029_{\text {syst }} \\
a_{2}= & -0.0432 \pm 0.0086_{\text {stat }} \pm 0.0034_{\text {syst }} & -0.0241 \pm 0.0129_{\text {stat }} \pm 0.0096_{\text {syst }} \\
\text { correlation } & 0.969 & -0.879
\end{array}
$$


The systematic errors quoted for the cusp results include the small external error as well (see Ref. [13]). No theoretical uncertainty is assigned yet as it is still under evaluation. In the $\mathrm{K}_{\mathrm{e} 4}$ case, the theoretical uncertainty contribution is even smaller than the experimental systematic error and thus has very little impact on the overall precision.

It is therefore interesting to combine the two analyzes to get a more precise result:

$$
\begin{gathered}
a_{0}=0.2210 \pm 0.0047_{\text {stat }} \pm 0.0015_{\text {syst }}, a_{2}=-0.0429 \pm 0.0044_{\text {stat }} \pm 0.0016_{\text {syst }} \\
a_{0}-a_{2}=0.2639 \pm 0.0020_{\text {stat }} \pm 0.0004_{\text {syst }}
\end{gathered}
$$

Figure 4 shows the two results and their combination in the planes $\left(a_{0}-a_{2}, a_{2}\right)$ and $\left(a_{0}, a_{2}\right)$, including statistical and systematic errors. The result $\left|a_{0}-a_{2}\right|$ obtained from the pionium life time measurement by the DIRAC collaboration [14] is also shown.
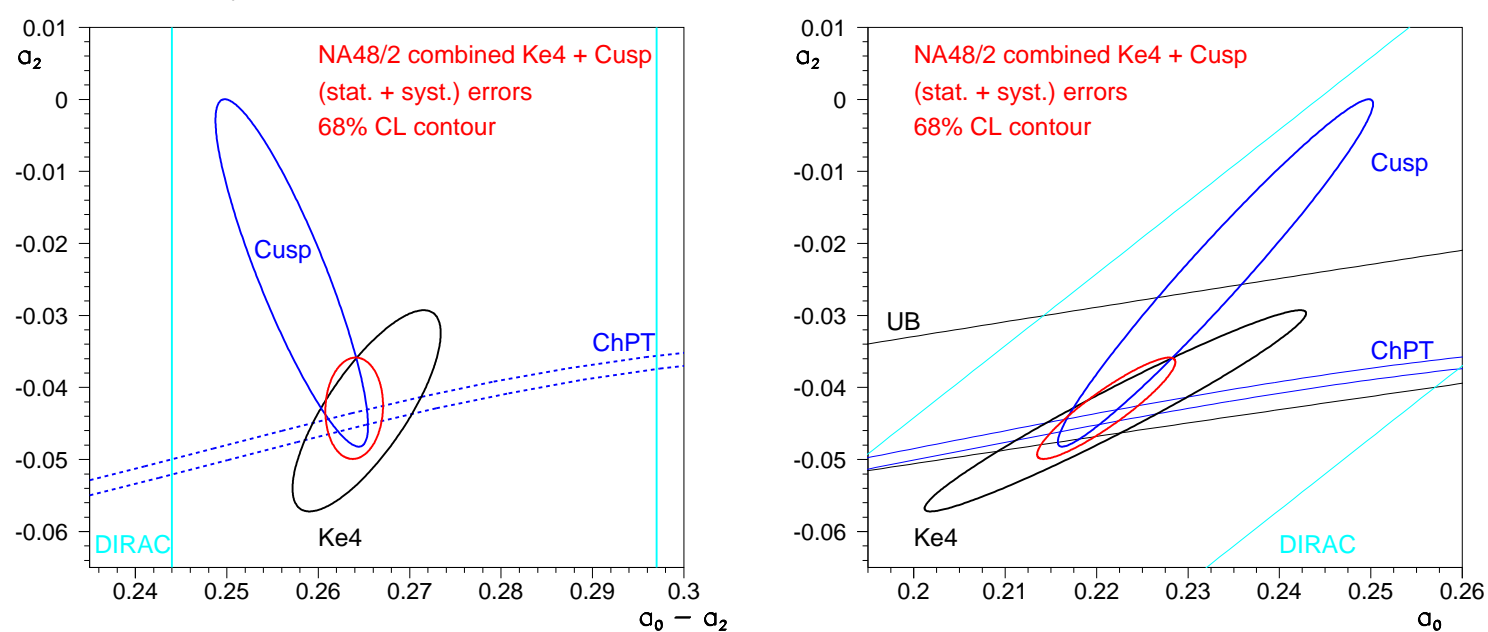

Figure 4: NA48/2 $\mathrm{K}_{\mathrm{e} 4}$ (black) and cusp (blue) results as 2-parameter fits in the $\left(a_{0}-a_{2}, a_{2}\right)$ (left) and $\left(a_{0}, a_{2}\right)$ (right) planes. In each plane the red contour corresponds to the combination of the results. The correlation coefficient is then 0.28 (resp. 0.91). The light blue lines correspond to the DIRAC result band.

\section{Summary}

Precise measurements have been obtained for the form factors and $\pi \pi$ phase shift in the analysis of 1.13 million $\mathrm{K}_{\mathrm{e} 4}$ decays. The observed variation of the phase shift with $M_{\pi \pi}$ allows to determine the scattering lengths $a_{0}$ and $a_{2}$ independently of ChPT prescriptions. The result is found in beautiful agreement with the predictions of ChPT once all isospin symmetry breaking effects are taken into account. The precise NA48/2 result dominates the combination with previous $\mathrm{K}_{\mathrm{e} 4}$ data (Figure $5 \mathrm{a}$ and $\mathrm{b}$ ). The combination of two independent measurements of the $\mathrm{S}$-wave scattering lengths by NA $48 / 2$ gives even more precise values of both $a_{0}$ and $a_{2}$ with similar \pm 0.005 (statistical + systematic) uncertainties. One should note that this is the first experimental determination ever obtained of $a_{2}$ with a $10 \%$ relative precision. The precise results from NA48/2 supersede previous experimental results and provide a powerful test of ChPT predictions as illustrated in Figure $5 \mathrm{k}$.

\section{Acknowledgments}

It is a pleasure to thank the scientific organizers and their local staff for the perfect running of the Workshop and their warmest hospitality in Bern. 

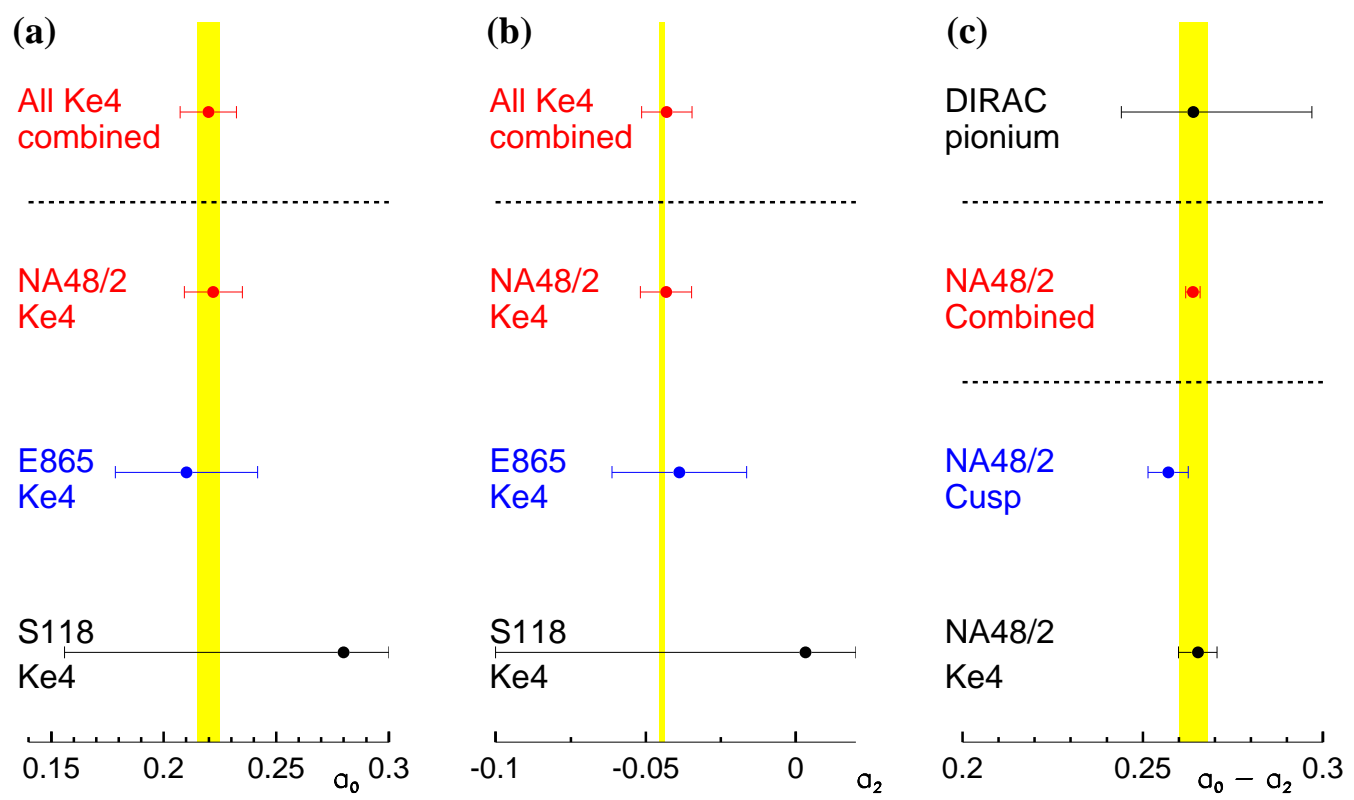

Figure 5: Experimental results for free $a_{0}$ (a) and $a_{2}$ (b) from each $\mathrm{K}_{\mathrm{e} 4}$ experiment and combined result dominated by NA48 precision. The right part of the large S118 error bar is truncated. (c): $a_{0}-a_{2}$ results from both Na48/2 analyzes and combined result. The DIRAC measurement is also shown. Color bands correspond to the best predictions from ChPT.

\section{References}

[1] NA48/2: J. Batley et al., Eur. Phys. J. C54 (2008) 411.

[2] E865: S. Pislak et al., Phys. Rev. Lett. 87 (2001) 221801, Phys. Rev. D67 (2003) 072004.

[3] S118: L. Rosselet et al., Phys. Rev. D15 (1977) 574.

[4] G. Colangelo, J. Gasser and A. Rusetsky, Eur. Phys. J. C59 (2009) 777.

[5] NA48/2: J. Batley et al., Phys. Lett. B649 (2007) 349.

[6] NA48: V. Fanti et al., Nucl. Instrum. Methods A574 (2007) 433.

[7] N. Cabibbo and A. Maksymowicz, Phys. Rev. 137 (1965) B438; Phys. Rev. 168 (1968) 1926.

[8] A. Pais and S. Treiman, Phys. Rev. 168 (1968) 1858.

[9] E. Barberio and Z. Was, PHOTOS, Comput. Phys. Commun. 79 (1994) 291.

[10] S. Roy, Phys. Lett. B36 (1971) 353.

[11] B. Ananthanarayan, G. Colangelo, J. Gasser, H. Leutwyler, Phys. Rep. 353 (2001) 207,

S. Descotes, N. Fuchs, L. Girlanda, J. Stern, Eur. Phys. J. C24 (2002) 469.

[12] G. Colangelo, J. Gasser, H. Leutwyler, Nucl. Phys. $B 603$ (2001) 125, Phys. Rev. Lett. 86 (2001) 5008.

[13] NA48/2: S. Giudici, this conference, PoS(CD09)002 , J. Batley et al., Phys. Lett. B633 (2006) 173.

[14] DIRAC: B. Adeva et al., Phys. Lett. B619 (2005) 50. 